\title{
SERUM PIGMENT EPITHELIUM DERIVED FACTOR AS A NEW MARKER IN THE METABOLIC SYNDROME
}

\author{
Jehan H Sabry, Osama S. El-Shaaer., Amr. M. El Hammady² \\ and Asmaa .A .El Fallah \\ ${ }^{1}$ Clinical and Chemical Pathology Department, Faculty of Medicine, Benha University, Egypt \\ ${ }^{2}$ Internal Medicine Department, Faculty of Medicine, Benha University, Egypt
}

DOI: http://dx.doi.org/10.24327/ijrsr.2017.0803.0021

\section{ARTICLE INFO}

\section{Article History:}

Received $15^{\text {th }}$ December, 2016

Received in revised form $25^{\text {th }}$

January, 2017

Accepted $23^{\text {rd }}$ February, 2017

Published online $28^{\text {th }}$ March, 2017

\section{Key Words:}

Metabolic syndrome, biomarker, pigment epithelium derived factor, Hs-CRP

\begin{abstract}
Background: Metabolic syndrome represents a cluster of related metabolic abnormalities, including central obesity, hyperglycemia, hypertension, dyslipidemia, and insulin resistance, with insulin resistance and central obesity in particular recognized as causative factors. Aim of the study: to determine serum level of Serum pigment epithelium derived factor (PEDF) and to examine whether PEDF serum level are associated with metabolic syndrome risk factor. Materials and Methods: The present study was conducted on 90 subjects subdivided into two groups. Patient group included 70 patients with metabolic syndrome attending the outpatient clinic in Benha University Hospital. and control group, this group included 20 apparently healthy individuals, Blood samples were drawn from all subjects to assess serum levels of PEDF, Hs-CRP, lipid profile, F.B.S, creatinine and uric acid. Results: PEDF level was significantly higher in patient group than control group, significant elevation of Hs-CRP, FBS, TG, LDL-cholesterol and uric acid in patient group versus control group and significant decrease of HDL-cholesterol in patient group versus control group. Significant positive correlation between PEDF (patient group) and each of the components of Metabolic syndromeas BMI,WC, LDL-cholesterol and total cholesterol, triglyceride, systolic \&diastolic blood pressure and the accumulation of the number of this component collectively. Conclusion: Based on the evidence presented within the study, PEDF correlate significantly with metabolic syndrome and could provide a minimally-invasive mean for early detection and specific treatment of this disorder. Further research is encouraged to determine the efficacy of applying this biomarker fordiagnosis of metabolic syndrome.
\end{abstract}

Copyright (C) Jehan H Sabry et al, 2017, this is an open-access article distributed under the terms of the Creative Commons Attribution License, which permits unrestricted use, distribution and reproduction in any medium, provided the original work is properly cited.

\section{INTRODUCTION}

Metabolic syndrome is a cluster of metabolic abnormalities which confers upon an individual a substantial increase in cardiovascular disease (CVD) risk - approximately twice as high as those without the syndrome. Compared to those without metabolic syndrome, those with it are at an increased risk of mortality from CVD, coronary heart disease, stroke, vascular dysfunction, and all-cause mortality ${ }^{(1)}$.

Several different organizations have outlined diagnostic criteria for metabolic syndrome, which designates values for obesity (waist circumference or BMI), triglyceride levels, HDL (High Density Lipoprotein) levels, hypertension, hyperglycemia, and sometimes urine albumin or albumin: creatinine ratio ${ }^{(2)}$ Regardless of which criteria are used, the primary concern is early detection of potential CVD complications and early intervention $^{(3,4) \text {. }}$
Though the NCEP ATP III report and WHO have both identified CVD as the primary clinical outcome of metabolic syndrome, most people with metabolic syndrome will have insulin resistance, which results in increased risk for type 2 diabetes. Once diabetes becomes clinically apparent, CVD risk rises sharply. In addition to CVD and type 2 diabetes, individuals with metabolic syndrome are seemingly more susceptible to other conditions, including polycystic ovary syndrome, fatty liver, cholesterol gallstones, asthma, sleep disturbances, and some forms of cancer, such as breast, pancreatic, colorectal, and prostate ${ }^{(5,6)}$.

Pigment epithelium-derived factor (PEDF) is an endogenously produced glycoprotein with a molecular weight of $50 \mathrm{kD}$, occurring commonly in various organs and exhibiting diverse biological activity ${ }^{(7)}$.It belongs to the super family of serine protease inhibitors acting like substrates rather than inhibitors of serine proteases. It is synthesized especially in the liver, but 
also in a wide range of human tissues such as the lung, brain, kidney, and adipose tissue ${ }^{(8)}$.

PEDF is a potent inhibitor of angiogenesis and shows differentiating activity in cells derived from retina and central nervous system and blocks fibrogenesis in diabetic kidneys through inhibition of transforming growth factor beta and connective tissue growth factor expression and function. Moreover, it inhibits endothelial cell injury in vitro suggesting the involvement of PEDF in atherosclerosis. It was discovered that PEDF levels are associated with metabolic syndrome and several coronary risk factors ${ }^{(9)}$. It is believed that PEDF has neuroprotectiveproperties ${ }^{(10)}$ acts as an antioxidant and antiinflammatory agent ${ }^{(11)}$ inhibits cellular proliferation and supports cell differentiation. ${ }^{(12)}$

\section{2-patient and methods}

The study was conducted since October 2012 to May 2014 at Department of Clinical and Chemical pathology, Faculty of medicine. Benha University, after approval by the Local Ethical Committee, the study was designed to include 90subjects subdivided into two groups. Patients' group: included 70 patients with metabolic syndrome attending the Outpatient Clinic of Diabetes and Metabolism in Benha University Hospital. They were 38 males and 32 females. And control group: this group included twenty apparently healthy individuals of matched age and sex. They were 9 females and 11 males. Patients with acute or chronic inflammation, liver disease, kidney disease and diabetic patients were excluded. All patients were subjected to full history laying stress on diet habits, drug intake, family history of obesity and use of tobacco and alcohol. Anthropometric measurements included: weight in kilograms, height in centimeters, body mass index $\left(\mathrm{kg} / \mathrm{m}^{2}\right)$ calculated by the formula: [BMI =Weight (in $\mathrm{kg}$ ) / Height (in meter $\left.{ }^{2}\right)$ and waist circumference in centimeters. Blood pressure (BP) was measured in supine position using an upright standard sphygmomanometer. Vigorous physical activity and smoking were avoided for at least $30 \mathrm{~min}$ before BP measurement.

Five milliliters of overnight fasting venous blood were withdrawn from each participant in the study under complete aseptic precautions into serum separator tubes. After coagulation, samples were centrifuged (at $1500 \mathrm{~g}$ for 15 minutes). The separated serum was divided into two aliquots. One was designated for the immediate assay biochemical markers. The other one was stored at $-20^{\circ} \mathrm{C}$ for subsequent assay of PEDF and HS-CRP. Hemolysed samples were discarded.

Laboratory investigations which included: lipids [total cholesterol, high-density lipoprotein (HDL) cholesterol and triglycerides] fasting blood glucose, creatinine and uric acid, were measured using commercially available kits using autoanalyzer (Biosystem BT 310 Barcelona- Spain). LDL-C was calculated using the Friedewald's equation ${ }^{(13) .}$

\section{Measurement of (PEDF) by ELISA}

PEDF was measured using BioVendor human ELISA kits provided by BioVendor- Laboratornímedicínaa.s. (Karasek, 1767/6211, 00 Brno, Czech Republic) Briefly:standards, quality controls and samples were incubated in micro titration wells coated with polyclonal anti-human PEDF antibody .After incubation and washing, biotin labeled polyclonal anti-human PEDF antibody was added and incubated with captured PEDF. After another washing, streptavidin-HRP conjugate was added. After incubation and the last washing step, Conjugate was allowed to react with the substrate solution (TMB).Reaction was stopped by addition of acidic solution and absorbance of the resulting yellow product was measured. The absorbance was proportional to the concentration of PEDF.A standard curve was constructed by plotting absorbance value against concentrations of standards, and concentrations of samples were determined using this standard curve ${ }^{(14)}$

\section{Measurement of (HS-CRP) by ELISA}

Highly sensitive CRP was measured using Accu-bind ELISA kits provided by Monbind Inc. lake fores, CA92630 USA.

The microtiter plate provided in this kit had been precoated with an antibody specific to CRP. Standards or samples were then added to the appropriate microtiter plate wells with a HRP-conjugated antibody preparation specific for CRP to each microplate well and incubated. Then a TMB (3,3',5,5'tetramethyl-benzidine) substrate solution was added to each well. Only those wells that contain CRP, HRP-conjugated antibody exhibited a change in color. The enzyme- substrate reaction was terminated by the addition of a sulphuric acid solution and the color change was measured spectrophotometrically at a wavelength of $450 \mathrm{~nm}$. The concentration of CRP in the samples was then determined by comparing the O.D. of the samples to the standard curve ${ }^{(15)}$.

\section{RESULTS}

Comparison between studied groups as regard anthropometric measures showedthat there was significant increase in $\mathrm{WC}$ and BMI in patients' group $(\mathrm{P}<0.001)$ (Fig1).

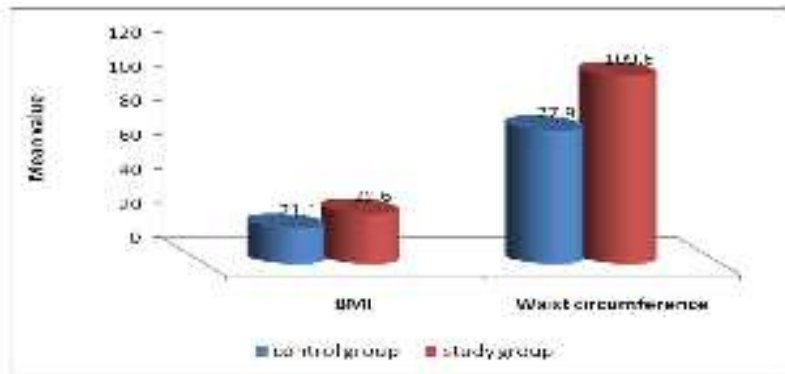

Figure 1 Comparison between studied groups as regard BMI $\left(\mathrm{Kg} / \mathrm{m}^{2}\right)$ andwaist circumference $(\mathrm{cm})$

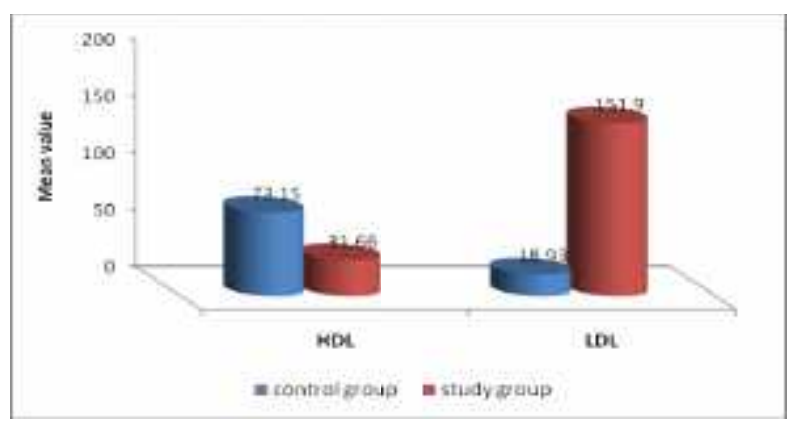

Figure 2 Comparison between studied groups as regard lipid profile $(\mathrm{mg} / \mathrm{dl})$

Blood pressure measurement revealed statistically significant increase in patients'group versus controlgroup $(\mathrm{P}<0.001)$. 
Laboratoryresults showed that there was statistically significant increase inboth fasting serum glucose and uric acid in patients'group versuscontrol group $(\mathrm{P}<0.001)$, while there was no significant difference between both groups regarding serum creatinine level.

Lipid profile analyses showed that there was statistically significant increase in the mean total cholesterol, triglycerides, LDL-cholesterol levels and statistically significant decrease in HDL cholesterol levels in patients' group versus control group $(\mathrm{P}<0.001)$ (Fig2).

PEDF was statistically significantly increased in patients' group $(56.38 \pm 10.78 \mu \mathrm{g} / \mathrm{ml})$ versuscontrol $(27.99 \pm 6.86 \mu \mathrm{g} / \mathrm{ml})$ group $(\mathrm{P}<0.001)$, showing significant positive correlations with both Hs-CRP and number of components of metabolic syndrome $(r=0.420, \mathrm{P}<0.001)$ in the patients' group (Fig 3,Fig 4) .

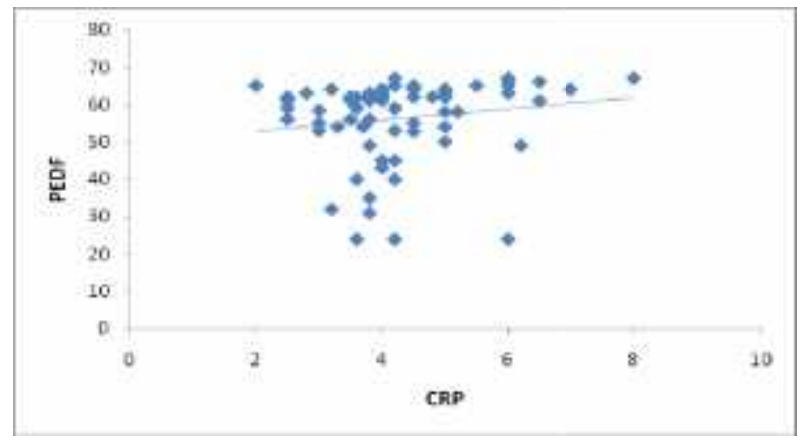

Figure3 Correlation between PEDF $(\mu \mathrm{g} / \mathrm{ml})$ and HS CRP(mg/L) among the patients' group

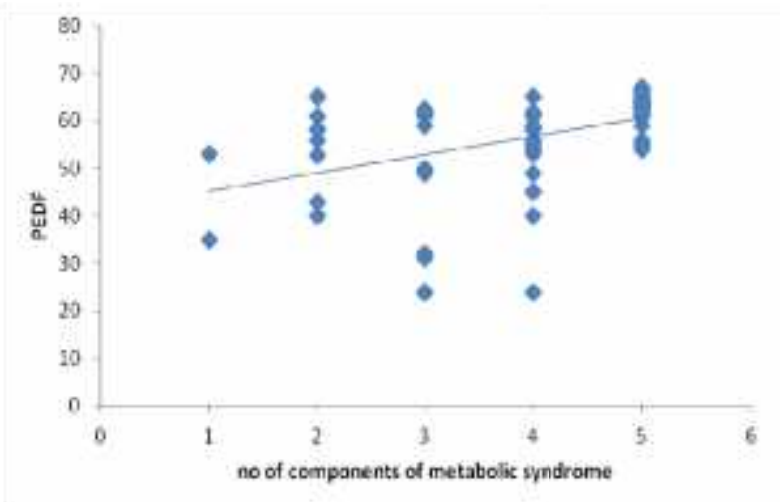

Figure 4 Correlation between PEDF and number of components of metabolic syndrome

Stepwise multiple regression analysis of PEDF with the risk factors of the metabolic syndrome showed that only HDL \&total cholesterol were significant independent determinants of serum PEDF levels, the remaining factors were not independently related to PEDF (Table1a). And it also shows that $\mathrm{PEDF}$ is a significant predictor of the number of components of metabolic syndrome (Table 1b)

ROC curve analysis showed that PEDF sensitivity and specificity for the presence of metabolic syndrome were $83.6 \%$ and $53.5 \%$ respectively at a cut-off value of $53.5 \mu \mathrm{g} / \mathrm{ml}(\mathrm{AUC}=$ $0.683,95 \%$ CI $0.43-0.83$ ) (Fig 5).
Table1 Stepwise multiple regression analysis of PEDF with the risk factors of the metabolic syndrome

Table (1a)

\begin{tabular}{|c|c|c|c|}
\hline \multirow{3}{*}{ Variables } & \multirow{2}{*}{$\begin{array}{l}\mathbf{R}^{2} \\
\mathbf{b}_{0} \\
\end{array}$} & \multicolumn{2}{|r|}{0.204} \\
\hline & & \multicolumn{2}{|r|}{58.7} \\
\hline & B & $\mathbf{P}$ & $95 \%$ CI of $\beta$ \\
\hline HDL $(\mathrm{mg} / \mathrm{dl})$ & -0.62 & $<0.01$ & {$[-1.7-(-1.6)]$} \\
\hline T.Cholesterol (mg/dl) & 0.08 & $<0.05$ & {$[0.02-0.14]$} \\
\hline
\end{tabular}

Table (1b)

\begin{tabular}{cccc}
\hline \multirow{2}{*}{ Variables } & R2 & & \multicolumn{2}{c}{$\mathbf{0 . 1 7 7}$} \\
\cline { 2 - 4 } & b0 & & $\mathbf{1 . 3}$ \\
\cline { 2 - 4 } & $\mathbf{B}$ & $\mathbf{P}$ & $\mathbf{9 5 \%} \mathbf{C I}$ of $\boldsymbol{\beta}$ \\
\hline $\mathrm{PEDF}(\mu \mathrm{g} / \mathrm{ml})$ & 0.046 & $<0.001$ & {$[0.022-0.07]$} \\
\hline
\end{tabular}

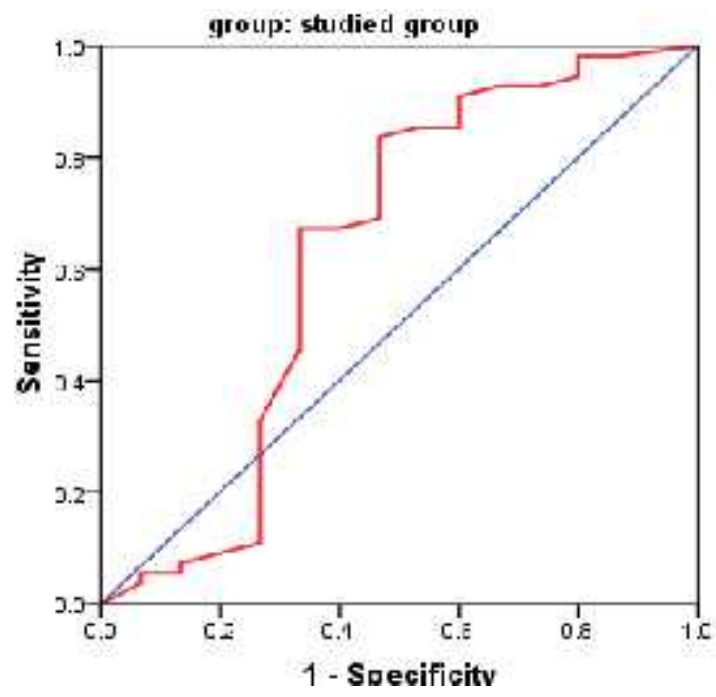

Figure 5 ROC curve analysis of PEDF and metabolic syndrome presence

\section{DISCUSSION}

Metabolic syndrome is a multifactorial condition that stems from obesity as the causative factor, though the exact mechanism is yet to be determined. Many suggest that oxidative stress, the hallmark of obesity, is linked to a chronic low-grade inflammation. The induced systemic oxidative stress is thought to be at least partly responsible for the dysregulated secretion of adipokines that contributes to metabolic syndrome (16)

A panel of biomarkers such as PEDF could be used clinically to help predict and establish metabolic syndrome in individuals would be of immense value, not only in treating those that already have the syndrome, but in decreasing the overall prevalence of the disease in the general population.

Liver is the major site of PEDF (Pigment Epithelium Derived Factor) synthesis. ${ }^{(17)}$ The mechanism of its action remains largely unclear. Antifibrogenic activity of intrahepatic PEDF has been demonstrated ${ }^{(18)}$. PEDF is secreted by fat cells as well, playing a role in the development of insulin resistance, metabolic syndrome, and liver steatosis ${ }^{(9,19-23)}$.

Results of this study revealed significant positive correlation between PEDF and each of BMI and WC. These findings go on line with another study performed by Stejskal et al. ${ }^{(21)}$ who concluded that PEDF is correlated with BMI and that PEDF is 
positively associated with obesity, particularly with visceral fat depot in Caucasian subjects.

According to the results of this study, correlation tests have revealed a positive correlation between PEDF and each of total cholesterol, LDL-cholesterol, and it was significant with triglyceride, On the other hand, there was a significant negative correlation with HDL-cholesterol. Thisresults was approved by Yamagishi et al. ${ }^{(9)}$ who found significant negative correlation between PEDF and HDL cholesterol and significant positive correlation with triglycerides. Also correlation tests have revealed a positive correlation between PEDF and fasting serum glucose levels.

In the setting of insulin resistance, increased flux of free fatty acids to the liver increases hepatic triglyceride synthesis. Thus, hypertriglyceridaemia is an excellent reflection of the insulin resistant condition, reduction of HDL is a consequence of changes in HDL composition and metabolism. In the presence of hypertriglyceridaemia, a decrease in the cholesterol content of HDL results from decreases in the cholesteryl ester content of the lipoprotein core with variable increases in triglyceride, in addition to HDL, the composition of LDL is also modified in a similar way. This change in LDL composition is attributable to relative depletion of unesterified and esterified cholesterol, and phospholipids, with either no change or an increase in LDL triglyceride ${ }^{(24)}$.

Regarding metabolic syndrome component and the accumulation of the number of co- mponents of metabolic syndrome $\&$ according to the results of this study, correlation tests have revealed a significant positive correlation between PEDF and the accumulation of number of metabolic syndrome components. This results were in agree with a study done by Yamagishi et al. ${ }^{(9)}$ andthe salient findings of the study was that PEDF levels were not only associated with the components of metabolic syndrome but also higher in proportion to the accumulation of the number of components.

The association of PEDF with MetS and its components has been verified in many previous studies (21) PEDF level was identified as an independent predictor for the development of MetS. ${ }^{(25)}$.

Our study showed a positive correlation between PEDF and each of systolic and diastolic blood pressure. This goes on line with Sabater et al. ${ }^{(26)}$ who found that the changes in circulating PEDF concentration were positively associated with the changes in systolic and diastolic blood pressure after controlling for the change in BMI, however, only the association with systolic blood pressure remained significant.

Previous studies concluded that plasma PEDF correlated significantly with metabolic parameters including BP, DM duration, HbA1c, triglycerides, high-density lipoprotein cholesterol, BMI, and WC as well as with hypertension/use of antihypertensive and use of lipid-lowering drugs ${ }^{(27)}$.

In a study by Kajikawa et $a l^{(28)}$ they concluded that serum level of PEDF was significantly correlated with body mass index, high-density lipoprotein cholesterol, glucose, and that it may be a factor directly associated with atherosclerosis Others also reported that plasma PEDF was positively associated with obesity indices and diabetic vascular complications. ${ }^{(29)}$
This was against a study made by Li et al $^{(30)}$ who investigate serum PEDF levels in GDM women there was no correlation between PEDF concentration with age, week of gestation, prepregnancy BMI, and blood lipids levels.

In the present study stepwise multiple regression analysis of PEDF with the risk factors of the metabolic syndrome showed that only HDL \&T.cholesterol were significant predictors of PEDF, the remaining factors were not independently related to PEDF, it also shows that PEDF is significant predictor of component of metabolic syndrome. In a study made by Stejskal et al. (21) multivariate analysis revealed that no measured parameters were significant independent determinants of PEDF serum level. Evaluation of test validity characters using ROC curve defined a moderate diagnostic yield of PEDF with sensitivity $83.6 \%$ and specificity $53.3 \%$ at a cut off value 53.5 $\mu \mathrm{g} / \mathrm{ml}$

Akin et al.$^{(31)}$ concluded that PEDF is increased in obese type 2 diabetic humans, so therapeutic strategies to inhibit PEDF action in muscle and liver or to prevent adipocyte PEDF release may represent a viable approach to ameliorate obesity-induced insulin resistance and its associated pathologies.

\section{CONCLUSION}

Metabolic syndrome is a condition with genetic and acquired etiologies that results in CVD complications in populations across the world given the rates of obesity, hypertension, and diabetes. PEDF level in blood is elevated in patients with metabolic syndrome with a known and predictable associations and can provide a useful mean to detect those at risk and intervene as needed. Depending on the available knowledge that PEDF possesses anti-oxidant and anti-inflammatory properties, serum PEDF levels may be elevated as a counter system in the metabolic syndome.

\section{Reference}

1. Ford ES. The metabolic syndrome and mortality from cardiovascular disease and all-causes: findings from the National Health and Nutrition Examination Survey II Mortality Study. Atherosclerosis. 2004; 173:309-14.

2. Aguilar M, Bhuket T, Torres S, Liu B, Wong RJ. Prevalence of the metabolic syndrome in the United States, 2003-2012. Jama. 2015; 313:1973-4.

3. Grundy SM, Cleeman JI, Daniels SR, Donato KA, Eckel RH, Franklin BA. et al. Diagnosis and management of the metabolic syndrome: an American Heart Association/National Heart, Lung, and Blood Institute scientific statement. Current opinion in cardiology. 2006; 21:1-6.

4. Alberti KG, Zimmet P, Shaw J. Metabolic syndrome-a new world-wide definition. A Consensus Statement from the International Diabetes Federation. Diabetic medicine: $a$ journal of the British Diabetic Association. 2006; 23:469-80.

5. Grundy SM, Brewer HB Jr, Cleeman JI, Smith SC Jr, Lenfant C, American Heart A. et al. Definition of metabolic syndrome: Report of the National Heart, Lung, and Blood Institute/American Heart Association conference on scientific issues related to definition. Circulation. 2004; 109:433-8. [PubMed] 
6. Bhandari R, Kelley GA, Hartley TA, Rockett IR. Metabolic syndrome is associated with increased breast cancer risk: a systematic review with metaanalysis. International journal of breast cancer. 2014; 2014:189384.

7. Petersen SV, Valnickova Z, Enghild JJ. Pigmentepithelium-derived factor (PEDF) occurs at a physiologically relevant concentration in human blood: Purification and characterization. Biochem J 2003; 374:199-206.

8. Matsuzawa Y (2006): Therapy in sight: Adipocytokines in metabolic Syndrome and related cardiovascular disease. Nat ClinPractCardiovasc Med 2006; 3:35-42.

9. Yamagishi S, Adachi H, Abe A, Yashiro T, Enomoto M, Furuki K, et al. Elevated serum levels of pigment epithelium-derived factor in the metabolic syndrome. $J$ ClinEndocrinolMetab 2006; 91:2447-50.

10. Bilak MM, Corse AM, Bilak SR, Lehar M, TombranTink J, Kuncl RW. Pigment epithelium-derived factor (PEDF) protects motor neurons from chronic glutamatemediated neurodegeneration. J NeuropatholExpNeurol 1999; 58:719-28.

11. Zhang SX, Wang JJ, Gao G, Shao C, Mott R, Ma JX. Pigment epithelium-derived factor (PEDF) is an endogenous anti-inflammatory factor. FASEB $J$ 2006;20:323-5.

12. Hoshina D, Abe R, Yamagishi SI, Shimizu H. The role of PEDF in tumor growth and metastasis. CurrMol Med. 2010;10:292-5

13. Friedewald WT, Levy RI, Fredrickson DS.Estimation of the concentration of low-density lipoprotein cholesterol in plasma, without use of the preparative ultracentrifuge. Clinical Chemistry. 1972; 18 (6): 499-502. PMID 4337382

14. Becerra, S. P.Structure-Function Relationships of PEDF.Exp Eye Res.2006; 82(5), 739-740

15. Macy, E.M. et al., Variability in the measurement of Creactive protein in healthy subjects: implications for reference interval and epidemiological applications. Clin. Chem.1997;43(1): 52-58 .

16. Furukawa S, Fujita T, Shimabukuro M, Iwaki M, Yamada Y, Nakajima Y, et al. Increased oxidative stress in obesity and its impact on metabolic syndrome. The Journal of clinical investigation. 2004; 114: 1752-61.

17. Tombran-Tink J, Mazuruk K, Rodriguez IR, Chung D, Linker T, Englander E, et al. Organization, evolutionary conservation, expression and unusual Alu density of the human gene for pigment epithelium-derived factor, a unique neurotropic serpin. Mol Vis 1996; 2:11.

18. Ho TC, Chen SL, Shih SC, Wu JY, Han WH, Cheng $\mathrm{HC}$, et al. Pigment epithelium-derived factor is an intrinsic antifibrosis factor targeting hepatic stellate cells. Am J Pathol 2010; 177:1798-811.

19. Famulla S, Lamers D, Hartwig S, Passlack W, Horrighs A, Cramer A, et al. Pigment epithelium-derived factor (PEDF) is one of the most abundant proteins secreted by human adipocytes and induces insulin resistance and inflammatory signaling in muscle and fat cells. Int $J$ Obes 2011;35:762-72.
20. Yilmaz Y, Eren F, Ayyildiz T, Colak Y, Kurt R, Senates $\mathrm{E}$, et al. Serum pigment epithelium-derived factor levels are increased in patients with biopsy-proven nonalcoholic fatty liver disease and independently associated with liver steatosis. ClinChimActa 2011; 412:2296-9.

21. Stejskal D, Karpísek M, Svesták M, Hejduk P, Sporová L, Kotolová H. Pigment epithelium-derived factor as a new marker of metabolic syndrome in Caucasian population. J Clin Lab Anal 2010; 24:17-9.

22. Borg ML, Andrews ZB, Duh EJ, Zechner R, Meikle PJ, Watt MJ. Pigment epithelium-derived factor regulates lipid metabolism via adipose triglyceride lipase. Diabetes 2011; 60:1458-66.

23. Chung C, Doll JA, Gattu AK, Shugrue C, Cornwell M, Fitchev $\mathrm{P}$, et al. Anti-angiogenic pigment epitheliumderived factor regulates hepatocyte triglyceride content through adipose triglyceride lipase (ATGL). J Hepatol 2008;48:471-8

24. Eckel R, Grundy S and Zimmet: The metabolic syndrome. The Lancet.2005; 365:1415-1428.

25. Chen C, Tso AW, Law LS, Cheung BM, Ong KL, Wat NM, Janus ED, Xu A, Lam KS: Plasma level of pigment epithelium-derived factor is independently associated with the development of the metabolic syndrome in Chinese men: a 10-year prospective study. $J$ ClinEndocrinolMetab 2010, 95:5074-5081.

26. Sabater M, Jose M and José F: Circulating Pigment Epithelium-Derived Factor Levels Are Associated with Insulin Resistance and Decrease after Weight Loss The Journal of Clinical Endocrinology \& Metabolism. 2010; $95:$ :(10) 4720-4728

27. HuiE, Yeung CY, Lee PCH., et al.: Elevated Circulating Pigment Epithelium-Derived Factor Predicts the Progression of Diabetic Nephropathy in Patients With Type 2 Diabetes. The journal of medical endocrinology and metabolism. 2014;99 (11): 21692177

28. KajikawaM, MaruhashiT, and IwamotoY et al.: Circulating level of pigment epithelium-derived factor is associated with vascular function and structure: A crosssectional study. 2016; 225: 91-95

29. NakamuraK, Yamagishi S, Adachi H, Kurita-Nakamura Y, Matsui T.Serum levels of pigment epitheliumderived factor (PEDF) are positively associated with visceral adiposity in Japanese patients with type 2 diabetes. Diabetes Metab Res Rev. 2009 ; 25 : 52 - 56

30. Li TH, Qiu CJ, Yu XJ,et al:: Increased Serum Pigment Epithelium-Derived Factor in Women with Gestational Diabetes Is Associated with Type 2 Diabetes. International Journal of Endocrinology. 2015; 2015, Article ID 346938, 5 pages.

31. Akın S, Yazgan D, ÇınarN ,et al.: Pigment epitheliumderived factor (PEDF) increases in type 2 diabetes after treatment with metformin. ClinEndocrinol. 2012; 77(6):852-856. 